\title{
Really, Disease Doesn't Matter? A Commentary on Correlates of Depressive Symptoms in Women Treated for Early-Stage Breast Cancer
}

\author{
Michael F. Scheier and Vicki S. Helgeson, Carnegie Mellon University, Pittsburgh, PA
}

Our initial response to the results reported by Bardwell et $\mathrm{al}^{1}$ was very odd-bordering on ironic. Here is an article that shows that objective, disease-related variables have virtually nothing to do with depressive symptoms following diagnosis and treatment for breast cancer. Rather, all of the action seems to be due to psychosocial factors, such as optimism and the lack of social support, or to the patient's subjective response to the disease and its treatment, as indexed by ratings of pain, physical functioning limitations, and symptoms. Our immediate reaction was to ask, how can this be? Surely biomedical factors have to have some impact.

So where is the irony? It derives from the fact that we are both psychologists. As such, we should have relished the findings that were reported. Yet, we were skeptical. We should be clear though about the source of our skepticism. It was not the fact that psychosocial factors were shown to be important. Of course, we knew those variables would matter. Our disbelief was in the fact that biomedical factors had virtually no effect.

Our commentary is divided into two parts. In the first part, driven largely by our skepticism, we describe a couple of alternative explanations that occurred to us that may have made the findings less than definitive. We also describe efforts to gather additional information that might speak to some of the concerns we have. As will become clear, the findings do seem to hold up to careful scrutiny. Accordingly, in the second part of the commentary, we describe some of the implications that the findings have, if presumed accurate.

One of our concerns had to do with the absence of an association between stage and depressive symptomatology. The problem had to do with homogeneity with respect to stage of disease. That is, the majority of women had stage I and II disease, and a small number had early stage III disease. The lack of relation between stage and depressive symptoms might have been due to the restricted range on this variable. However, one of us has data on a group of women ( $\mathrm{N}=284$; unpublished) diagnosed with stage I, II, and IV breast cancer. Forty percent of the women had stage I, 30\% had stage II, and 30\% had stage IV. Depressive symptoms were measured by a 10 -item version ${ }^{2}$ of the Center for Epidemiologic Studies Depression Scale. ${ }^{3}$ The correlation between disease stage and depressive symptoms in this sample was not close to being significant $(r=-0.04)$. We were surprised by this finding. Still, the data helped to convince us that restriction of range does not provide a viable interpretation of why there was no association between disease stage and depressive symptoms in the findings reported by Bardwell et al. ${ }^{1}$ The absence of an association in fact seems real.

A second concern had to do with the amount of time that passed since the women were diagnosed and treated. Is it possible that the lack of a relation between objective characteristics of the disease and depressive symptoms in this sample were due to the fact that patients were too distant from the initial diagnosis? Might a relation have appeared among patients who were newly diagnosed? Bardwell et al ${ }^{1}$ found no relation of time since diagnosis to depression, but patients in their study were diagnosed as much as 4 years earlier with the earliest group having been diagnosed during the past year. Perhaps associations between biomedical factors and depressive symptoms are very short lived and dissipate even within a 1-year period.

Again, one of us has data on a large group of women $(\mathrm{N}=312$; unpublished) with largely stage I (25\%) and II (69\%) disease who were more recently diagnosed with breast cancer (average diagnoses within 3 months) and found no relation of stage of disease, kind of surgery, or estrogen receptor status to depressive symptoms. In fact, in one article, we reported that stage of disease did not distinguish among different trajectories of mental functioning over a 4 -year period. ${ }^{4}$ Thus, stronger relations do not emerge among newly diagnosed women, suggesting that this was not a factor in the results reported.

We have offered two alternative explanations for the absence of associations reported by Bardwell et al, ${ }^{1}$ both of which were ruled out by examination of other data sets that were available. There are several other questions that remain that we could not resolve with recourse to additional data, and we would like to at least mention them in passing. First, although it seems clear that there are no simple associations between biomedical factors and depressive symptoms, more complex associations may exist. In this regard, it is possible that there is a synergistic relation between objective disease characteristics and psychosocial variables in predicting mental health outcomes. For example, optimism or previous life events might be more strongly related to depression among patients with more severe disease, as might be implied by a stress-diathesis model. Such a model would assume that biomedical factors are important in increasing the impact of psychosocial factors, as stress increases and passes some threshold as one moves from early to advanced disease. This possibility was not explored by Bardwell et al. ${ }^{1}$

Our final point concerns the manner in which treatment effects were evaluated. Specifically, although the authors examined 
the relation of different treatment combinations to mental health, they did not examine the relation of individual treatments to outcomes. That is, although representative of the general classes of treatment women with breast cancer face, the categories of treatment used in this study may have been too crude to detect effects. There are a variety of kinds and strengths of chemotherapy protocols in use that one would expect to have different effects on physical functioning. It might be better in future work to separate these treatment categories into more refined groupings to see whether differences in associations emerge when different chemotherapy protocols are taken into account.

As noted, we were initially skeptical of the findings, but became more and more convinced as we thought about the article and explored associations in other data sets that biomedical factors really do not predict depressive symptoms. Given our (perhaps still somewhat guarded) conversion, we move to the second part of our commentary. Specifically, given that we accept what is reported by Bardwell et $\mathrm{al}^{1}$ at face value, what are the clinical implications of the findings? One implication is that health care professionals should not assume that everyone will respond the same way to a given disease or given stage of disease. There will be patients who are diagnosed with advanced disease who are resilient due to personality or due to a history of life experiences that has enabled them to cope with the current trauma in a healthy way. In other words, all people with advanced disease are not necessarily in need of psychosocial assistance. Similarly, one cannot assume that someone who has been diagnosed with a very early and easily treatable disease will respond with a mild level of distress that dissipates with time.

When recruiting participants into one of our early interventions, we can recall a patient with stage I disease who initially declined the intervention because she told us her physician had said she had treatable disease, should not be distressed, and did not need any assistance. Fortunately, she ultimately declined to take his advice, participated in the randomized trial, and ended up being assigned to a psychosocial group intervention from which she greatly benefited. As with any other traumatic event, a diagnosis of cancer needs to be placed in context. There may be other factors in a patient's life, like whether they have support available and whether they are facing other life traumas, that may be more determinant of how they respond to their disease than whether the disease is classified as stage I or stage III or stage IV. In brief, attending physicians should not assume that someone with ductal carcinoma in situ is not in need of psychosocial intervention and someone with stage IV has to have it. The data from Bardwell et al ${ }^{1}$ document that you cannot predict level of depressive symptomatology from extensiveness of disease.

A second implication is that psychosocial interventions should be targeted at specific groups of patients based on their psychosocial profile rather than objective characteristics of the disease. A cognitive-behavioral intervention might benefit pessimistic patients regardless of the source of their cancer (lung $v$ breast), the stage of their disease, and the treatment they are receiving. A support intervention might benefit patients who lack supportive resources in their natural environment, again regardless of the objective characteristics of their disease. It should be much easier to identify a psychosocial intervention to target a psychosocial characteristic than a diverse group of patients who just happen to have the same kind of, stage of, and treatment for cancer.

Finally, although objective characteristics of the disease did not predict depression in the final multivariate regression equation, physical functioning and physical symptoms did. These physical limitations, however, do not arise from a vacuum. They most likely arose from the impact of the disease and its treatment, albeit independently from the severity of the disease and nature of the treatment. Perhaps those expecting associations between biomedical predictors and depressive symptoms should take some solace in this observation. The disease may in fact be having some impact on depressive symptoms, but only indirectly as reflected through the patient's reactions to it. Still, it seems to be a very good day to be a psychologist.

\section{(C) 2006 by American Society of Clinical Oncology}

\section{REFERENCES}

1. Bardwell, WA, Natarajan, L, Dimsdale, JE, et al: Objective cancer-related variables are not associated with depressive symptoms in women treated for early-stage breast cancer. J Clin Oncol 24:2420-2427, 2006

2. Andresen EM, Malmgren JA, Carter WB, et al: Screening for depression in well older adults: Evaluation of a short form of the CES-D (Center for Epidemiologic Studies Depression Scale). Am J Prev Med 10:77-84, 1994

3. Radloff LS: The CES-D scale: A self-report depression scale for research in the general population. Appl Psychol Measurement 1:385-401, 1977

4. Helgeson VS, Snyder PR, Seltman H: Psychological and physical adjustment to breast cancer over 4 years: Identifying distinct trajectories of change. Health Psychol 23:3-15, 2004

\section{Acknowledgment}

This editorial was supported by funds awarded to the Pittsburgh Mind-Body Center at the University of Pittsburgh and Carnegie Mellon University (NIH HL076852 and HL076858) and by NIH Grants No. CA64711 and CA104078. We would like to thank Karen A. Matthews for providing a most helpful commentary on our commentary.

\section{Authors' Disclosures of Potential Conflicts of Interest}

The authors indicated no potential conflicts of interest.

\section{Author Contributions}

Conception and design: Michael F. Scheier, Vicki S. Helgeson

Collection and assembly of data: Michael F. Scheier, Vicki S. Helgeson

Data analysis and interpretation: Michael F. Scheier, Vicki S. Helgeson

Manuscript writing: Michael F. Scheier, Vicki S. Helgeson

Final approval of manuscript: Michael F. Scheier, Vicki S. Helgeson 